\title{
Downlink Power Control Algorithms for Cellular Radio Systems
}

\author{
Tsern-Huei Lee, Member, IEEE Jen-Cheng Lin, and Yu T. Su, Member, IEEE
}

\begin{abstract}
Power control is an effective technique to reduce cochannel interference and increase capacity for cellular radio systems. Optimum centralized power control can minimize the outage probability, but requires the information of all link gains in real time, which is very difficult to successfully implement for a large system; besides, the computational complexity of an optimum power control algorithm makes it impractical for real implementations. In this paper, we propose some centralized power control algorithms with reasonable computational complexity. One of the algorithms, called the SMIRA algorithm, has an outage probability that is very close to the minimum. We also study a class of distributed power control algorithms that can achieve a balanced carrier-to-interference ratio with probability one. Among the class of algorithms, we found that the one proposed in [6] gives the minimum outage probability.
\end{abstract}

\section{INTRODUCTION}

$\mathbf{F}$ REQUENCY REUSE IS the core concept in cellular mobile systems because it can significantly increase system capacity. The interference due to common use of the same channel sets a limit on frequency reuse. Therefore, it is important to reduce the cochannel interference in designing a high capacity cellular radio system. Power control is a technique that can be used to reduce cochannel interference and allow as many receivers as possible to obtain satisfactory reception. Reception is said to be satisfactory if the carrierto-interference ratio (CIR) is greater than the minimum CIR required or the system protection ratio.

Aein [1] proposed controlling transmitter power to achieve a balanced CIR. Nettleton and Alavi [2] showed that CIR balancing can improve the capacity of a spread spectrum cellular mobile radio system. Under the balancing scheme, all receivers experience the same CIR. Unfortunately, it is possible that for a balanced CIR the reception may be unsatisfactory for all receivers. To achieve satisfactory reception, some transmitters may need to be prohibited from transmitting. Zander [4] studied this problem and derived an optimum power control scheme that minimizes the outage probability. The outage probability is defined as the probability of having a CIR smaller than the system protection ratio on a given link. However, the computational complexity makes the optimum power control scheme impractical. In [4], Zander proposed

Manuscript received February 3, 1994; revised July 19, 1994. This work was supported in part by the National Science Council, Republic of China, under Contract NSC 81-0404-E-009-527.

T. H. Lee and Y. T. Su are with the Department of Communication Engineering. National Chiao Tung University, Hsinchu, Taiwan 300, Republic of China.

J.-C. Lin is with the Institute of Electronics, National Chiao Tung University, Hsinchu. Taiwan 300, Republic of China.

IEEE Log Number 9407336. a simple procedure, called the stepwise removal algorithm (SRA), for practical implementations. The SRA algorithm, step by step, removes one cell until the required CIR is achieved in the remaining cells.

The power control algorithms studied in [4] are centralized and require knowledge of all the active link gains that may not be possible to acquire in real time for large systems. For this reason, Zander [5] proposed a distributed balancing algorithm that uses only local CIR information. It was shown that the distributed algorithm can achieve CIR balancing with probability one and thus, when combined with cell removal algorithms, can obtain a minimum outage probability. However, the convergence speed of that algorithm is not satisfactory. If the allowed maximum number of iterations is small, then the distributed balancing algorithm may result in an outage probability much greater than the optimum value. Another distributed power control algorithm that converges faster than Zander's algorithm was proposed in [6].

In this paper we study both centralized and distributed power control algorithms. Some new centralized power control algorithms that take into account both transmitting power and the link gains are proposed. Numerical results show that, compared with the SRA algorithm, the new schemes achieve a smaller outage probability with only a negligible increase in computational complexity. We also study a class of distributed power control algorithms that includes the algorithms proposed in [5] and [6] as special cases. The class of algorithms can achieve CIR balancing with probability one. From numerical examples we found that the algorithm proposed in [6] gives the least outage probability among the investigated class of algorithms.

The system model is described in Section II. Sections III and IV consider respectively centralized and distributed power control algorithms. In Section V, some numerical examples are provided. Conclusions are finally drawn in Section VI.

\section{SYSTEM MODEL}

The cellular system investigated in this paper consists of a finite number of cells. Cells using the same channel are placed symmetrically in a hexagonal grid. Base stations use omnidirectional antennas and are located at the center of the cells. The locations of the mobiles are assumed to be uniformly distributed over the cell area. Each cell has $M$ independent channel pairs, each consisting of independent uplink and downlink channels. In this paper, we consider power control algorithms for downlink channels. 
For simplicity, the adjacent channel interference is neglected. The thermal noise sources are also neglected. The set of cells using a certain channel at some given instant is called the cochannel set and the size of the cochannel set is denoted by $N$. All cochannels are assumed to be in use. The total interference power is modeled as the sum of the powers of all active interferers and the transmission quality is assumed to depend only on CIR. Under these assumptions, the CIR at the mobile in cell $i$ is given by

$$
\begin{aligned}
C I R_{i} & =\frac{R_{i}}{\sum_{j=1}^{N} R_{j}-R_{i}}=\frac{T_{i} L_{i i}}{\sum_{j=1}^{N} T_{j} L_{i j}-T_{i} L_{i i}} \\
& =\frac{T_{i}}{\sum_{j=1}^{N} T_{j} Z_{i j}-T_{i}}
\end{aligned}
$$

where $R_{j}$ is the power received from the $j$ th transmitter, $T_{j}$ is the transmitting power used by the base station in cell $j, L_{i j}$ is the gain on the link between the mobile in cell $i$ and the base station in cell $j$ at some given moment, and $Z_{i j}=\frac{L_{i j}}{L_{i j}}$ is the normalized link gain. It is clear that $Z_{i i}=1$ for all $i, 1 \leq i \leq N$. Moreover, the value of $L_{i j}$ is assumed to be a constant (and needs to be measured for the centralized power control algorithms). The assumption is reasonable if the power control algorithm can converge in a short period. The link gain $L_{i j}$ is modeled as

$$
L_{i j}=\frac{A_{i j}}{d_{i j}^{\nu}}
$$

where $A_{i j}$ is the attenuation factor, $d_{i j}$ is the distance between the mobile in cell $i$ and the base station in cell $j$, and $\nu$ is a constant that models the large scale propagation loss. The attenuation factor models the power variation due to shadowing. We assume that $A_{i j}, 1 \leq i, j \leq N$, are independent, $\log$ normal, identically distributed, random variables with $0 \mathrm{~dB}$ expectation and $\sigma \mathrm{dB} \log$ variance. The parameter value of $\sigma$ in the range of $4-10 \mathrm{~dB}$ and the propagation constant $\nu$ in the range of 3-5 usually provide good models for urban propagation [7].

Let $\mathrm{CIR}_{0}$ be the system protection ratio or the minimum CIR required for satisfactory reception. The outage probability, denoted by $P_{\text {outage }}$, is defined as

$$
P_{\text {outage }}=\frac{1}{N} \sum_{j=1}^{N} P_{r}\left\{\mathrm{CIR}_{j}<\mathrm{CIR}_{0}\right\}
$$

where $\mathrm{CIR}_{j}$ is the carrier-to-interference ratio at the mobile in cell $j$. A CIR is said to be achievable in the cochannel set if there exists a power vector $\mathbf{T}=\left[T_{1}, T_{2}, \cdots, T_{N}\right]^{T}$ such that $\mathrm{CIR}_{i} \geq \mathrm{CIR}$ for all $i, 1 \leq i \leq N$. It was shown [5] that the maximum achievable CIR, denoted by $\mathrm{CIR}^{*}$, is given by

$$
\mathrm{CIR}^{*}=\frac{1}{\lambda^{*}-1}
$$

where $\lambda^{*}$ is the largest real eigenvalue of the positive link gain matrix $\mathbf{Z}=\left[Z_{i j}\right]$. Moreover, the power vector $\mathbf{T}^{*}$ achieving this maximum is the eigenvector of $\mathbf{Z}$ corresponding to the eigenvalue $\lambda^{*}$. All mobiles experience the same CIR* when $\mathbf{T}^{*}$ is used.

\section{Centralized Power Control Algorithms}

For centralized power control, it is assumed that the link gain matrix $\mathbf{Z}$ is available. Therefore, the maximum achievable CIR* can be obtained, because $\lambda^{*}$ can be determined. As a consequence, optimum power control is possible. An optimum power control algorithm (PCA) will, by removing as few cells as possible, find the largest submatrix of $\mathbf{Z}$ for which $\mathrm{CIR}_{0}$ is achievable. The following brute force algorithm is an optimum PCA.

Algorithm 1: Brute Force Algorithm (BFA) [4]:

Step 1: Determine $\mathrm{CIR}^{*}$ corresponding to $\mathrm{Z}$. If $\mathrm{CIR}^{*} \geq$ $\mathrm{CIR}_{0}$, then use the eigenvector $\mathbf{T}^{*}$ and stop; otherwise perform Step 2.

Step 2: Remove all combinations of at most $N-2$ cells and compute the eigenvalues of each reduced system. If for some $i, 1 \leq i \leq N-2, \mathrm{CIR}^{*} \geq C I R_{0}$, then use the corresponding eigenvector and stop; otherwise remove $N-1$ cells and stop.

Notice that when using the BFA algorithm to remove any combination of $n$ cells $(1 \leq n \leq N-2)$, one needs to determine the eigenvalue and eigenvector pair $\left(\begin{array}{l}N \\ n\end{array}\right)=\frac{N !}{n !(N-n) !}$ times. Therefore, in the worst case, one has to determine the eigenvalue and eigenvector pair $\left(\begin{array}{c}N \\ 1\end{array}\right)+\left(\begin{array}{c}N \\ 2\end{array}\right)+\cdots+$ $\left(\begin{array}{c}N \\ N-2\end{array}\right)=2^{N}-N-2$ times to remove $N-1$ cells to achieve $\mathrm{CIR}_{0}$. The computational complexity involved in the BFA algorithm makes it impractical because the link gains are likely to change before the algorithm settles. For this reason, Zander proposed the following stepwise removal algorithm for practical implementations.

Algorithm 2: Stepwise Removal Algorithm (SRA) [4]:

Step 1: Determine $\mathrm{CIR}^{*}$ corresponding to $\mathbf{Z}$. If $\mathrm{CIR}^{*} \geq$ $\mathrm{CIR}_{0}$, then use the eigenvector $\mathbf{T}^{*}$ and stop; otherwise set $N^{\prime}=N$ and perform Step 2 .

Step 2: Remove cell $k$ for which the maximum of the row and column sums, $R_{k}=\sum_{j=1}^{N^{\prime}} Z_{k j}$ and $R_{k}^{T}=\sum_{j=1}^{N^{\prime}} Z_{j k}$, is maximized and form the $\left(N^{\prime}-1\right) *\left(N^{\prime}-1\right)$ matrix $\mathbf{Z}^{\prime}$. Determine CIR* corresponding to $\mathbf{Z}^{\prime}$. If $\mathrm{CIR}^{*} \geq \mathrm{CIR}_{0}$, then use the corresponding eigenvector and stop; otherwise set $N^{\prime}=N^{\prime}-1$ and repeat Step 2 .

For the SRA algorithm, to remove one cell, one needs to find the eigenvalue and eigenvector pair once. So, in the worst case, one needs to solve the eigen system $N-2$ times that is far less than the $2^{N}-N-2$ times required for the BFA algorithm. However, the SRA algorithm need not be an optimum PCA. In Step 2 of the SRA algorithm, only the link gain matrix is used when a cell is to be removed. Improvement is possible if the transmitting power is also taken into consideration. To improve the performance of the SRA algorithm, we propose the following algorithm.

Algorithm 3: Stepwise Maximum-Interference Removal Algorithm (SMIRA): The SMIRA algorithm considers transmitting power to be an important factor in removing cells. The idea is that the larger the transmitting power, the greater the interference it causes to mobiles in the other cells. Therefore, if a cell which uses a high transmitting power is removed, then it is likely that the remaining cells can achieve $\mathrm{CIR}_{0}$. 
The SMIRA algorithm is described below.

Step 1: Determine $\mathrm{CIR}^{*}$ corresponding to $\mathrm{Z}$. If $\mathrm{CIR}^{*} \geq$ $\mathrm{CIR}_{0}$, then use the eigenvector $\mathrm{T}^{*}$ and stop; otherwise set $N^{\prime}=N$ and perform Step 2 .

Step 2: Compute $R_{l}=\sum_{j=1, j \neq l}^{N^{\prime}} T_{j} * Z_{l j}, R_{l}^{T}=T_{l} *$ $\sum_{j=1, j \neq l}^{N^{\prime}} Z_{j l}$, and $R_{\max , l}=\max \left(R_{l}, R_{l}^{T}\right)$ for all $l, 1 \leq$ $l \leq N^{\prime}$. Remove cell $k$ for which $R_{\max , k}=\max _{l} R_{\max , l}$ and form the $\left(N^{\prime}-1\right) *\left(N^{\prime}-1\right)$ matrix $\mathbf{Z}^{\prime}$. Determine $\mathrm{CIR}^{*}$ corresponding to $\mathrm{Z}^{\prime}$. If $\mathrm{CIR}^{*} \geq \mathrm{CIR}_{0}$, then use the corresponding eigenvector and stop; otherwise set $N^{\prime}=$ $N^{\prime}-1$ and repeat Step 2.

The term "maximum interference" is used because $R_{l}$ represents the total interference received by the mobile in cell $l$ and $R_{l}^{T}$ represents the total interference to other mobiles caused by base station $l$. Clearly, the SMIRA algorithm requires $N^{\prime 2}$ more multiplications than the SRA algorithm each time a cell is removed. However, the computational complexity is dominated by solving the eigen system and thus the $N^{\prime 2}$ multiplications are negligible.

Two other power control algorithms-- one of which considers the received interference, the other the transmitted interference-are listed below. Notice that the SMRIRA and the SMIRA algorithms have roughly the same computational complexity, as do the SMTIRA and the SRA algorithms. Algorithm 4: Stepwise Maximum Received Interference Removal Algorithm (SMRIRA): The SMRIRA algorithm is the same as the SMIRA algorithm except that $R_{\max , k}=\max _{l} R_{l}$. Algorithm 5: Stepwise Maximum Transmitted Interference Removal Algorithm (SMTIRA): The SMTIRA algorithm is the same as the SMIRA algorithm except that $R_{\max , k}=\max _{l} R_{l}^{T}$.

The outage probabilities of the above PCA's will be compared in section $\mathrm{V}$.

\section{DisTRIBUTED PCA'S}

For the centralized power control algorithms to be useful, one needs to measure all link gains in real time, which is impractical for a large cellular system. In [5], Zander proposed a distributed balancing algorithm in which each cell determines its next transmitting power based on the measured CIR in its own cell. The distributed balancing algorithm can be described as follows:

$$
\begin{aligned}
\mathbf{T}^{(0)} & =\mathbf{T}_{0} . \quad \mathbf{T}_{0}>\mathbf{0} \\
T_{i}^{(k+1)} & =\beta T_{i}^{(k)}\left(1+\frac{1}{\operatorname{CIR}_{i}^{(k)}}\right), \quad \beta>0
\end{aligned}
$$

where $\mathbf{T}_{0}$ is an arbitrary positive vector, $T_{i}^{(k)}$ denotes the transmitting power of the base station in cell $i$ in the $k$ th discrete time, $\operatorname{CIR}_{i}^{(k)}$ denotes the CIR at the mobile in cell $i$ in the $k$ th discrete time, and $\beta$ denotes the weighting factor. Another distributed power control algorithm proposed in [6] adapts the transmitting power according to the observed interference, i.e.,

$$
T_{i}^{(k+1)}=\beta I_{i}^{\left(k^{\prime}\right)}
$$

where

$$
I_{i}^{(k)}=\sum_{j=1}^{N} T_{j}^{(k)} Z_{i j}-T_{i}^{(k)}
$$

is the interference power received by the mobile in cell $i$ in the $k$ th discrete time.

The above two distributed power control algorithms were both proved to be able to achieve CIR balancing with probability one. Moreover, it was found that the scheme proposed in [6] converges much faster than the Zander's scheme. In this paper, we study the following class of distributed power control algorithms.

$$
\begin{aligned}
& \text { A. A Class of Distributed Balancing Algorithms } \\
& \qquad \begin{aligned}
\mathbf{T}^{(0)}= & \mathbf{T}_{0}, \quad \mathbf{T}_{0}>\mathbf{0} \\
T_{i}^{(k+1)} & =\beta T_{i}^{(k)}\left((1-\alpha)+\frac{1}{\mathbf{C I R}_{i}^{(k)}}\right), \\
& \beta>0 \text { and }-\infty<\alpha \leq 1 .
\end{aligned}
\end{aligned}
$$

It is clear that $\alpha=0$ and $\alpha=1$ correspond, respectively, to the algorithms proposed in [5] and [6]. From (5), we get

$$
T_{i}^{(k+1)}=\beta\left(\sum_{j=1}^{N} T_{j}^{(k)} Z_{i j}-\alpha T_{i}^{(k)}\right)
$$

which when expressed in vector form is given by

$$
\mathbf{T}^{(k+1)}=\beta(\mathbf{Z}-\alpha \mathbf{I}) \mathbf{T}^{(k)}=\beta \mathbf{Y} \mathbf{T}^{(k)}
$$

where $\mathbf{Y}=\mathbf{Z}-\alpha \mathbf{I}$ is an irreducible nonnegative matrix [9]. Notice that $\mathbf{Y}$ is irreducible and nonnegative because all the link gains are positive real numbers. With the Perron-Frobenius Theorem [9], one can show that the above class of algorithms achieve CIR balancing with probability one. The proof is similar to those presented in [5] and [6] and thus is omitted.

Notice that when $\alpha$ approaches $-\infty$ one gets the fix power algorithm (i.e., without power control). Numerical examples presented in the following section show that $\alpha=1$ provides the best outage probability among the class of algorithms. In the numerical examples, the limited information stepwise removal algorithm (LI-SRA) proposed by Zander is adopted. The LI-SRA algorithm is described below.

LI-SRA Algorithm:

Step 1: [Initialize] Set $\mathbf{T}_{0}=\mathbf{1}$. Measure and store the CIR vector, $\operatorname{CIR}^{(0)}$.

Step 2: [Check CIR] If $\mathrm{CIR}_{i}^{(0)} \geq \mathrm{CIR}_{0}$ for all $i$, then stop; otherwise perform Step 3 .

Step 3: [Use PCA] Operate the distributed balancing algorithm (for a given $\alpha$ ) for at most $L$ steps. If at some step $k(k<L), \mathrm{CIR}_{i}^{(k)} \geq \mathrm{CIR}_{0}$ for all $i$, then stop; otherwise perform Step 4.

Step 4: [Remove one cell] Remove the cell that has the smallest initial CIR, $\mathrm{CIR}_{i}^{(0)}$. Go to Step 1. 


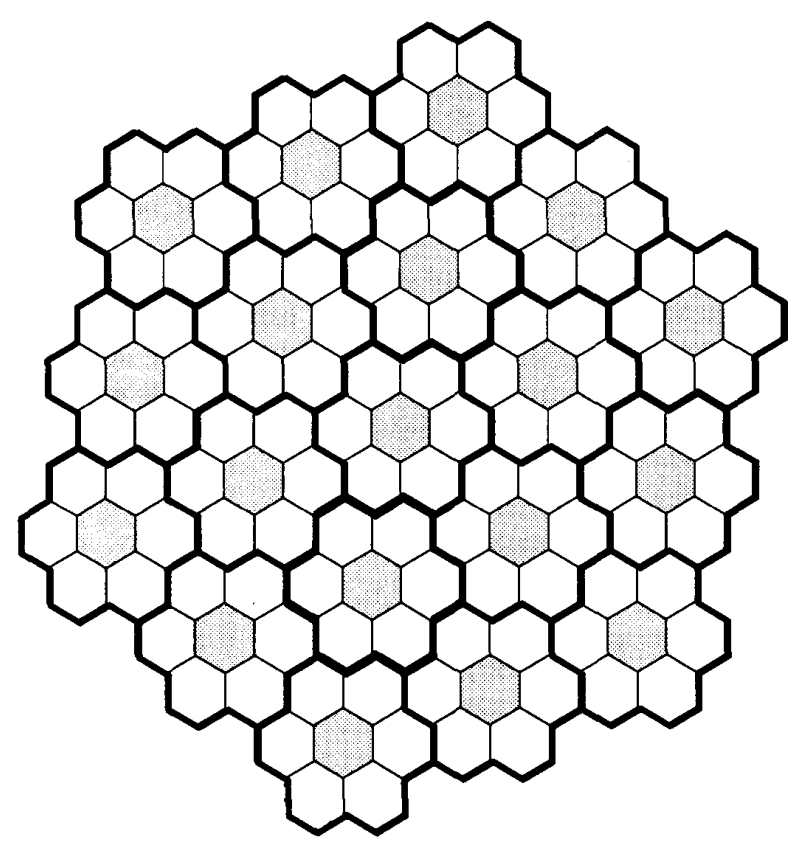

Fig. 1. 19 cochannel cells with seven-cell cluster.

\section{Numerical Results}

Numerical results were obtained for a system containing 19 cochannel cells that uses a seven-cell cluster, Fig. 1. All the values were obtained by means of Monte Carlo simulation for 500 independent configurations. In our study, we used $\sigma=6$ and $\nu=4$. Outage probability is measured over all 19 cochannels.

Consider centralized power control algorithms first. Fig. 2 shows the maximum achievable CIR after a number of cells were removed using various power control algorithms. It can be seen that the SMIRA algorithm is almost as good as the BFA algorithm and is consistently better than the other nonoptimum algorithms. The SMRIRA and the SMTIRA algorithms have roughly the same performance and are better than the SRA algorithm. The SMTIRA algorithm may become worse than the SRA algorithm for a large protection ratio. Fig. 3 compares the outage probabilities of these algorithms. Among the nonoptimum PCA's, the SMIRA algorithm gives the lowest outage probability. The improvement of SMIRA over SRA can be as high as $22 \%$ when the protection ratio is around $15 \mathrm{~dB}$. When only one cell is removed, our examples show that the SMIRA algorithm removes the cell in an optimum way with probability $64.8 \%$ (324 out of 500 ), whereas the SMRIRA, SMTIRA, and SRA algorithms achieve probabilities $52 \%$ ( 260 out of 500 ), $45.8 \%$ (229 out of 500 ), and $44 \%$ (220 out of 500 ), respectively. When two cells are removed, the probabilities for the SMIRA, SMRIRA, SMTIRA, and SRA algorithms are $48.8 \%, 31.2 \%, 25.4 \%$, and $22.2 \%$, respectively.

In Table I, we compare the ensemble mean of the maximum achievable CIR for these algorithms. The difference between the SMIRA algorithm and the BFA algorithm is only $1.55 \%$ or $1.69 \%$, when one cell or two cells are removed, respectively.

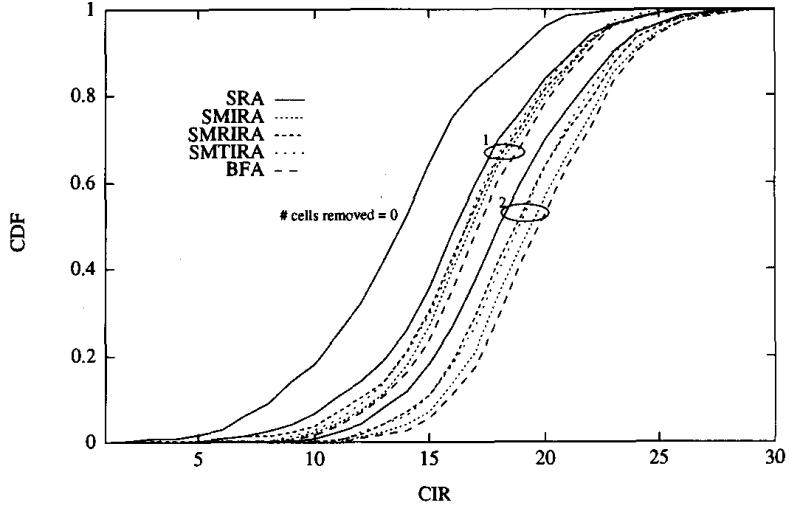

Fig. 2. Distribution of maximum achievable CIR for SRA and SMIRA.

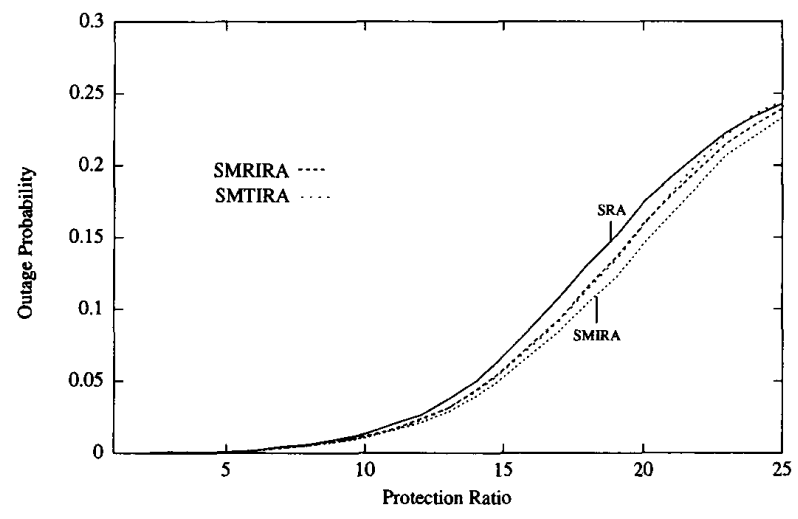

Fig. 3. Outage probability comparison among different algorithms.

TABLE I

Maximum ACHiEvable CIR

\begin{tabular}{|c|c|c|c|c|c|}
\hline $\begin{array}{c}\text { CIR algorithm } \\
\text { \# } \begin{array}{c}\text { cells } \\
\text { removed }\end{array}\end{array}$ & BFA & SRA & SMIRA & SMRIRA & SMTIRA \\
\hline 1 & 17.2911 & 16.1601 & 17.0237 & 16.6832 & 16.7217 \\
\hline 2 & 19.9138 & 18.2384 & 19.5767 & 18.9706 & 18.9566 \\
\hline
\end{tabular}

Therefore, considering the computational complexity and the outage probability, the SMIRA algorithm seems to be a good choice.

Let us consider now the distributed power control algorithms. From numerical results, we found that the ratio of $\left|\lambda_{j}-\alpha\right|\left(\lambda_{j}\right.$ is chosen to satisfy $\left|\lambda_{j}-\alpha\right| \geq\left|\lambda_{k}-\alpha\right|$ for all $k, 2 \leq k \leq N)$ to $\left|\lambda_{1}-\alpha\right|$, which determines the speed of convergence and thus the outage probability is a decreasing function of $\alpha$. Besides, the ratio for $\alpha=1$ is much smaller than the ratio for $\alpha=0$. Table II lists some ratios for $-1 \leq \alpha \leq 1$.

Figs. 4 and 5 show, respectively, an example of the variation of $\mathrm{CIR}_{i}, 1 \leq i \leq 19$, for $\alpha=0$ and $\alpha=1$ up to 25 iterations. In Fig. 4, CIR balancing cannot be achieved in 25 iterations. In Fig. 5, CIR $i$ converges to $17.35 \mathrm{~dB}$ in about 20 iterations for all $i$. If the protection ratio is $15 \mathrm{~dB}$, then at least one cell is removed for the algorithm with $\alpha=0$. 
TABLE II

Convergence Speed Comparison

\begin{tabular}{|c|c|c|c|c|c|c|c|}
\hline$\alpha$ & -1.0 & -0.9 & -0.8 & -0.7 & -0.6 & -0.5 & -0.4 \\
\hline$\frac{\left|\lambda_{j}-\alpha\right|}{\left|\lambda_{1}-\alpha\right|}$ & 0.9927 & 0.9923 & 0.9919 & 0.9914 & 0.9909 & 0.9903 & 0.9896 \\
\hline$\alpha$ & -0.3 & -0.2 & -0.1 & 0 & 0.1 & 0.2 & 0.3 \\
\hline$\frac{\left|\lambda_{j}-\alpha\right|}{\left|\lambda_{1}-\alpha\right|}$ & 0.9889 & 0.9880 & 0.9869 & 0.9857 & 0.9842 & 0.9823 & 0.9800 \\
\hline$\alpha$ & 0.4 & 0.5 & 0.6 & 0.7 & 0.8 & 0.9 & 1.0 \\
\hline$* \frac{\left|\lambda_{j}-\alpha\right|}{\left|\lambda_{1}-\alpha\right|}$ & 0.9769 & 0.9727 & 0.9665 & 0.9568 & 0.9391 & 0.8953 & 0.7938 \\
\hline
\end{tabular}

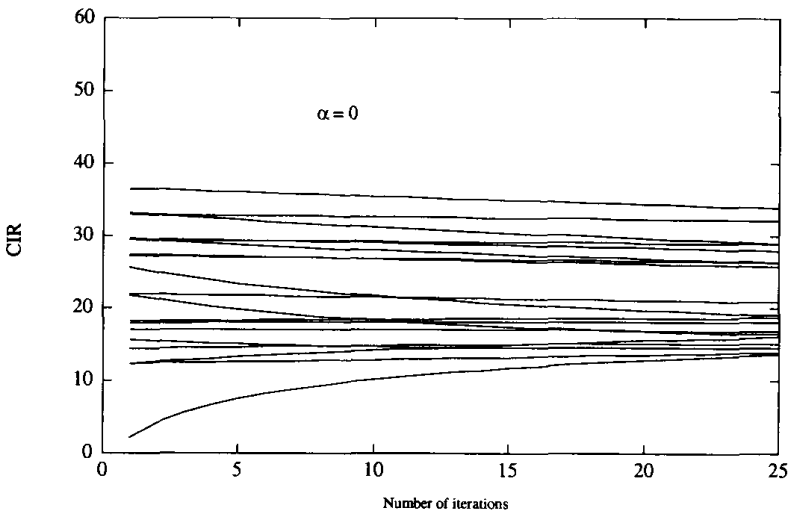

Fig. 4. CIR variation with $a=0$.

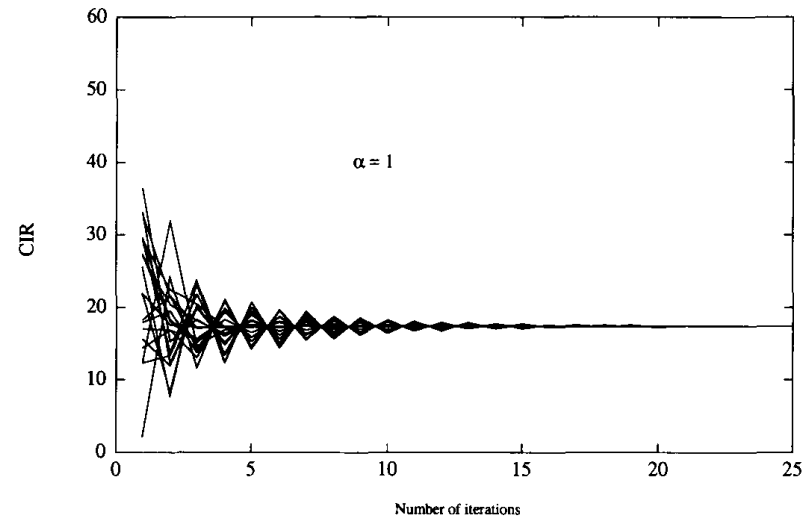

Fig. 5. CIR variation with $n=1$.

In Fig. 6, we compare the outage probabilities for the two algorithms with $\alpha=0$ and $\alpha=1$ for $L=8$ and 30. One can see clearly that the algorithm with $\alpha=1$ has a much better performance than the algorithm with $\alpha=0$. The outage probability of the algorithm with $\alpha=0$ is approaching that without power control when the protection ratio is high. On the other hand, the outage probability of the algorithm with $\alpha=1$ is close to that of the SMIRA algorithm, which has

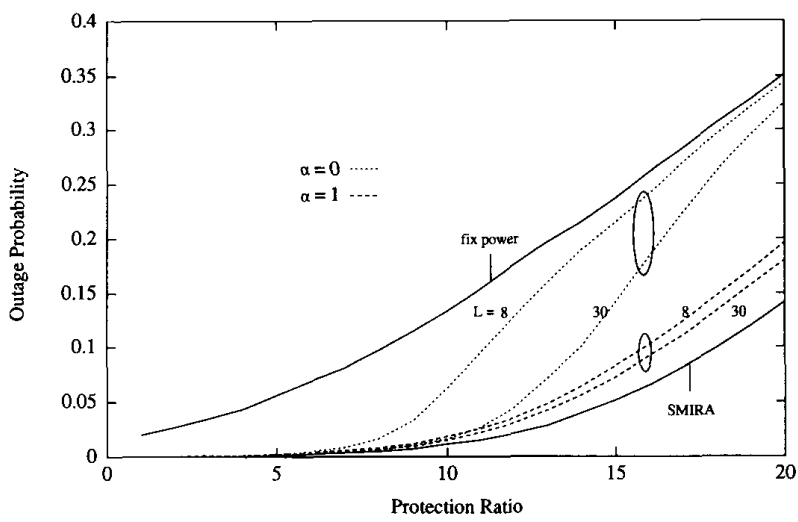

Fig. 6. Outage probability at different iterative steps.

been shown to be almost as good as the optimum power control algorithm. Therefore, for a large cellular system in which centralized power control is not feasible, the distributed balancing algorithm with $\alpha=1$ is a good scheme to use.

\section{CONCLUSION}

In this paper we have investigated both centralized and distributed power control algorithms for cellular radio systems. We showed that the SMIRA algorithm is a good centralized power control algorithm because its performance is very close to that of the optimum power control scheme and its computational complexity is much smaller than that of the BFA algorithm. A class of distributed power control algorithms which can achieve CIR balancing with probability one was studied. Among the algorithms in the class, we found that the scheme with $\alpha=1$, which was also studied in [6], results in the smallest outage probability. Some techniques can be used to further reduce the outage probability. For example, in Step 4 of the LI-SRA algorithm, one can remove the cell with maximum received interference of the final iteration $I_{i}^{(L)}$ rather than the cell with minimum initial CIR. However, the improvement is not very significant and thus is not presented.

\section{ACKNOWLEDGMENT}

The authors wish to thank Dr. Jens Zander and an anonymous referee for their valuable comments, which led to improvements in this paper.

\section{REFERENCES}

[1] J. M. Aein, "Power balancing in systems employing frequency reuse," COMSAT Tech. Rev., vol. 3, no. 2, Fall 1973.

[2] H. Alavi and R. W. Nettleton, "Downstream power control for a spread spectrum cellular mobile radio system," in Conf. Rec. GLOBECOM' 82 , Miami, FL, Nov. 29-Dec. 3, 1982.

13] T. Nagatsu, T. Tsuruhara, and M. Sakamoto, "Transmitter power control for cellular land mobile radio," in Proc. IEEE Vehicular Technol. Conf., 1985, pp. $12-15$.

[4] J. Zander, "Performance of optimum transmitter power control in cellular radio systems," IEEE Trans. Vehicular Technol., vol. 41, pp. 57-62, February 1992.

[5] J. Zander, "Distributed cochannel interference control in cellular radio systems," IEEE Trans. Veh. Technol., vol. 41, Aug. 1992. 
[6] S. A. Grandhi, R. Vijayan, and D. J. Goodman, "A distributed algorithm for power control in cellular radio systems," in Proc. 30th Allerton Conf. Commun.. Control and Computing, Monticello, IL, Sept./Oct. 1992.

[7] W. C. Y. Lee, "Elements of cellular mobile radio," IEEE Trans. Veh. Technol, vol. VT-35, pp. 48-56, 1986

[8] H. Hammnda, J. McGeehan, and A. Bateman, "Spectral efficiency of cellular land mobile radio systems," in Proc. 38th IEEE Veh. Technol. Conf., June 1988, pp. 616-620.

[9] F. R. Gantmacher, The Theory of Matrices. New York: Chelsea, 1974 vol. 2, ch. XIII.

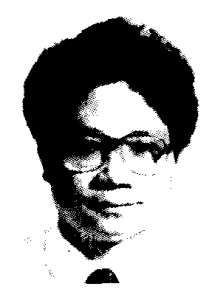

Tsern-Huei Lee (S'86-M'87) received the B.S. degree from National Taiwan University, Taipei, Taiwan, Republic of China, in 1981, the M.S. degree from the University of California, Santa Barbara, in 1984, and the Ph.D. degree from the University of Southern California, Los Angeles, in 1987, all in electrical engineering.

Since 1987 , he has been a member of the faculty of National Chiao Tung University, Hsinchu, Taiwan, Republic of China, where he is a Professor of the Department of Communication Engineering and a member of Center for Telecommunications Research. His current research interests are in wireless communications, communication protocols, broadband switching networks, and network flow control.

Dr. Lee received an Outstanding Paper Award from the Institute of Chinese Engineers in 1991.

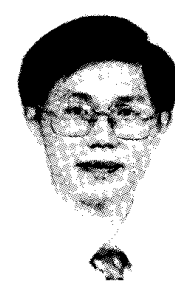

Jen-Cheng $L$ in received the B.S. degree in electronics engineering from Chung Yuan University, Chungli, Taiwan, Republic of China, in 1979, the M.S. degree in computer science \& information engineering from National Chiao Tung University, Hsinchu, Taiwan, in 1990. Currently, he is working toward the Ph.D. degree in electronics engineering at National Chiao Tung University, Hsinchu, Taiwan

He joined ERSO/ITRI, Hsinchu, Taiwan in 1983 , where he primarily worked on the development of device drivers as a project leader. In 1986 he was with New Development Corporation, Hsinchu, Taiwan. In 1987 he was with Link Communications Inc., Hsinchu, Taiwan, where he worked on the development of statistical multiplexer and VSAT system as a Project Manager. From September 1990 to March 1993, he served as a technical consultant to the Microelectronics Technology Inc., Hsinchu, Taiwan on maritime communications system and personal communications system. His current research interests include wireless communication networks and satellite communication systems.

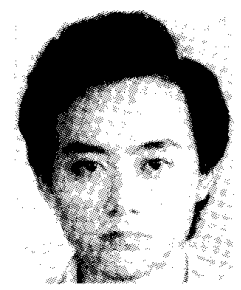

Yu T. Su (S'81-M'83) received the B.S.E.E. degree from Tatung Institute of Technology, Taiwan, in 1974, and the M.S. and Ph.D. degrees from the University of Southern California in 1983.

From May 1983 to September 1989 he was with LinCom Corporation, Los Angles, CA. He is currently an Associate Professor in the Department of Communication Engineering and the Microelectronic and Information Systems Research Center at the Chiao Tung University, Taiwan. Dr. Su's present research interests include communication theory and statistical signal processing. 